\title{
El capital social. Potencial para la investigación-acción de un paradigma emergente
}

\author{
Social capital: potential for action research of an emerging paradigm \\ José Manuel FERNÁNDEZ FERNÁNDEZ \\ Universidad Complutense de Madrid \\ jmfernan@cps.ucm.es
}

Quizá el ejemplo más importante de capital social en acción, cuando faltan los mecanismos formales de seguridad $y$ los instrumentos financieros sea el uso de las conexiones sociales por los pobres como principal medio de protección contra los riesgos y vulnerabilidades Woolcock, 2000, p. 19

Recibido: 15/03/2012

Revisado: 21/03/2012

Aceptado: 27/03/2012

Disponible on line: 03/08/2012

\section{Resumen}

El uso del término capital social ha crecido de modo exponencial en las últimas décadas con el riesgo de que se diluyan sus significados más relevantes para el análisis y la intervención social. En este artículo se presenta un análisis crítico del uso que hicieron de esta noción los científicos sociales que, desde posiciones teóricas muy diferentes, más han contribuyeron a su desarrollo actual. Se argumenta que aún no constituye un paradigma unificado. Para ilustrar su potencial para el análisis y la intervención social se ha seleccionado el modelo elaborado por Woolcock para el de desarrollo de comunidades pobres.

Palabras clave: comunidad, desarrollo, desigualdad, estructura, capital, redes, recursos, relaciones, vínculo.

\begin{abstract}
The use of the term «social capital» has increased exponentially during the recent decades to the point at which its relevant meanings risk being ruined for social analysis and intervention. In this paper, a critical evaluation is made of the applications of this idea by social scientists that, from many different theoretical perspectives, have contributed to its current development. It is argued that it has not attained the status of a unified paradigm. As an example of its potential for social analysis and intervention, the model worked out by Woolcock for the development of poor communities has been chosen. Key words: community, development, inequality, structure, capital, networks, resources, relations, link.
\end{abstract}

Referencia normalizada: Fernández Fernández, J.M. (2012): «El capital social. Potencial para la investigación-acción de un paradigma diferente». Cuadernos de Trabajo Social, 25(2): 297-308.

Sumario: Introducción. Un mismo término para muchos significados. 1. Las redes sociales como una forma de capital. La conceptualización de Bourdieu. 2. La controvertida fuerza de los vínculos débiles. 3. Contribución del capital social al desarrollo del capital humano. La teoría normativista de Coleman. 4. Algunos puntos débiles del la noción de capital social. El modelo de Portes y Sensenbrenner. 5. Capital social, sentido cívico y participación ciudadana. La teoría de Putnam. 6. El capital social como instrumento de desarrollo. La síntesis de Woolcock. 7. Desigualdad y capital social. 8. Referencias bibliográficas.

\section{Introducción. Un mismo término para mu- chos significados}

El concepto de capital social ha cautivado la imaginación y atención de un amplio rango de académicos y profesionales en diversas disciplinas y escenarios prácticos. Durante las tres últi- mas décadas se ha convertido en una de las aportaciones más populares de la teoría sociológica al lenguaje de la vida cotidiana para designar de modo simplificado las consecuencias positivas de la sociabilidad (Portes, 1998). Pero la diversidad de usos de una noción que ha gene- 
rado múltiples definiciones, conceptualizaciones y medidas empíricas, aún no integradas en un paradigma unificado, corre el riesgo de diluir su significado original y su potencial heurístico de concepto científico para la investigación de las diferentes cuestiones sociales a las que se está aplicando (Fukerson y Thomson, 2008).

Muchas de las controversias que han rodeado el tema del capital social tienen que ver con su amplio espectro de aplicación de diferentes tipos de problemas y su empleo en teorías que se basan en diferentes niveles de análisis. El capital social llegó a definirse como una fuente de control social, de beneficios mediados por la familia y de recursos mediados por redes no familiares. Esta ampliación del contenido del capital social, iniciada por Robert Putnam, permitió hablar de reserva de capital social comunitario e incluso nacional y de sus efectos estructurales. Progresivamente parece haberse producido cierta convergencia en el uso de ese término para referirse principalmente a recursos a los que se accede en las redes sociales, sentido que había estado desde el principio en la definición de Bourdieu (Lin, 2003). Sin embargo, en el debate actual sobre el tema hallamos dos enfoques diferentes, el normativo y el de los recursos En el primer campo hallamos autores como Coleman y Putnam. En el segundo estarían Bourdieu, Portes y Lin.

El enfoque normativo se mueve dentro de la tradición durkheimiana, analiza el capital desde la perspectiva de la organización social y enfatiza las transacciones de reciprocidad y la interiorización de valores. Muchos de los que se sitúan en este campo ven el capital social como una explicación universal de los patrones de desarrollo. Estos patrones se comprenden en términos de niveles variables de normas de confianza, de cohesión y solidaridad o de otros aspectos que conducen a la acción colectiva dentro de las regiones y estados-nación.

El enfoque del capital social como recurso se mueve en el marco de las tradiciones interaccionista y del conflicto, lo considera como un factor explicativo de los patrones de desigualdad en la acumulación de poder, prestigio y otras formas de desigualdad. Para quienes siguen esta orientación, un agente social puede utilizar sus redes sociales con la expectativa de conseguir algún tipo de beneficio. Como recurso, el capital social es convertible en otras formas.

\section{Las redes sociales como una forma de ca- pital. La conceptualización de Bourdieu}

El concepto de capital social desarrollado por Pierre Bourdieu es de los que más han contribuido a la gran difusión actual de esta noción en las ciencias sociales y a la reintegración de los análisis sociales y económicos (Smart, 1993, p. 388). De acuerdo con Alejandro Portes, «el primer análisis sistemático contemporáneo del capital social» y «posiblemente el teóricamente más refinado» fue el de Pierre Bourdieu» (Portes, 1998, p. 1).

La noción de capital construida por Bourdieu constituye una de las tres categorías centrales de su teoría de la práctica: campo, capital y habitus. Se trata de tres conceptos relacionales e intrínsecamente relacionados en su construcción teórica. El capital en sentido amplio lo definió como «una fuerza o energía dentro de un campo» o, de modo más parecido a Marx, como «trabajo acumulado». El capital puede adquirir diferentes formas, principalmente cuatro: capital económico, capital cultural, capital social y capital simbólico (Bourdieu, 1980, 1986). Estas formas diferentes de capital son convertibles entre sí. Esta convertibilidad sigue diferentes lógicas, según sea el tipo de capital, el campo en el que éste resulte más eficaz y el campo en el que se produzca el intercambio. Según Bourdieu, los diferentes tipos de capital pueden derivarse del capital económico, pero sólo al coste de un esfuerzo más o menos grande de transformación. Mientras que el capital económico es la forma más objetivable de capital, el capital social, que designa cosas inherentes a relaciones sociales concretas, como obligaciones y confianza es, por el contrario, su forma más volátil. Lo define como:

El conjunto de recursos actuales o potenciales que están vinculados a la posesión de una red durable de relaciones de mutuo conocimiento y reconocimiento más o menos institucionalizada; o, en otros términos, a la pertenencia a un grupo como conjunto de agentes que no sólo están dotados de propiedades comunes sino también unidos por vínculos permanentes y útiles (1980, p. 2).

Estos vínculos se fundan en intercambios inseparablemente materiales y simbólicos, por lo que no pueden reducirse a las relaciones objetivas de proximidad. 
De acuerdo con esa definición, el capital social es un recurso de los individuos que tiene su origen en el ámbito de las relaciones sociales. El volumen del capital social que posee un agente social no depende de cualidades individuales, sino de «la extensión de las redes de relaciones que puede movilizar con eficacia y del volumen del capital (económico, cultural o simbólico) poseído por cada uno de aquellos con los que se relaciona» (idem). El diferente volumen de capital social que poseen los individuos permite obtener rendimientos muy desiguales de un volumen de capital económico o cultural similar, según el grado en que pueden movilizar el capital de un grupo constituido como tal (familia, antiguos alumnos de escuelas de élite, nobleza, etc.) y más o menos provisto de capital (1980).

Para Bourdieu, el capital social depende, pues, del tamaño de las conexiones, del volumen o cantidad de capital que se encuentra en estas conexiones y de la capacidad de movilizarlas. Es un activo colectivo, producido y compartido por los miembros de un grupo definido, con claras fronteras, obligaciones de intercambio y reconocimiento mutuo. Ello supone repetidos intercambios que refuerzan el reconocimiento mutuo y los límites para afirmar y reafirmar el carácter colectivo del capital y la demanda que cada miembro hace del mismo. Como sucede con otras formas de capital, la acumulación de capital social por los individuos o los grupos, también necesita una inversión de recursos económicos y culturales. El desarrollo de redes de relaciones requiere estrategias de inversión social, consciente o inconscientemente orientadas hacia la construcción o reproducción de relaciones sociales directamente utilizables como recursos para obtener otros beneficios. «El rendimiento de este trabajo de acumulación es tanto mayor cuanto más importante es este capital» (p. 3).

El proceso de generación de capital social fue analizado con gran agudeza por Bourdieu (1989) en su investigación sobre las estrategias de producción y reproducción de las clases dominantes francesas. Las instituciones académicas de élite o, en el caso de sus vástagos menos aventajados, las «escuelas refugio», las familias y las grandes empresas son tres ámbitos privilegiados para la producción de un capital social que refuerza la posición y el privilegio de la alta burguesía. El capital social representa un proceso por el que los individuos de la clase dominante, por el conocimiento y reconocimiento mutuo, refuerzan y reproducen un grupo privilegiado que posee varias formas de capital (económico, cultural y simbólico).

\section{La controvertida fuerza de los vínculos débiles}

Otro eslabón importante en el desarrollo actual de la noción de capital social fueron los estudios de algunos economistas, como Glen Loury o Ben-Porath y y sociólogos, como Mark Granovetter o Nan Lin, que destacaron la importancia de las conexiones sociales en la explicación de las oportunidades de empleo y estratificación social.

Granovetter (1973, 1983) acuñó la expresión «fuerza de los vínculos débiles» para referirse al poder de la influencia indirecta de los lazos fuera del círculo de la familia y amigos en la obtención de empleo. Basándose en los datos de una investigación empírica que sugerían que quienes disponen de canales interpersonales consiguen empleos más satisfactorios y mejor remunerados, Granovetter (1974) elaboró una teoría de red sobre el flujo de información. Su hipótesis de «la fuerza de los vínculos débiles» establece que los vínculos más débiles tienden a formar puentes que enlazan a los individuos a otros círculos sociales para conseguir información útil que no suele estar disponible en sus propios círculos. Casi dos décadas después, Burt (1992) partió de la idea de Granovetter para desarrollar el concepto de structural holes, según el cual el capital social se basa en la relativa escasez de vínculos red, más que en su densidad.

Esa posición era contraria a la que mantuvieron Nan Lin, Walter Ensel y John Vaughn en Social Resources and Strenth of Ties (1981) y que puede etiquetarse «la fuerza de los vínculos fuertes», en contraste con Granovetter y Burt, y puede verse en otras áreas de la literatura sobre redes y movilidad social (Portes, 1998, pp. 1415). En realidad, Granovetter nunca sugiere que el acceso a vínculos más bien débiles que fuertes tenga como resultado la obtención de empleos de estatus más elevado. Las pistas sobre la conexión entre la fuerza de los vínculos y la consecución de estatus llegó indirectamente de un estudio realizado en Nueva York (Lin, Day- 
ton y Greenwald, 1978) que abrió el camino a la teoría de los recursos sociales de Nan Lin. Esta teoría parte de una imagen piramidal de la estructura macrosocial como posiciones jerarquizadas de acuerdo con ciertos recursos normativamente valorados como la riqueza, el estatus y el poder. En estas condiciones, la mejor estrategia para una acción instrumental que persiga mejorar el estatus dentro de la estructura social sería conseguir contactos más altos (Lin, 2003, 2008).

La investigación sobre las relaciones entre recursos sociales y logro de estatus examina dos procesos: el acceso al capital social y la movilización del capital social en el proceso de consecución de estatus. La investigación ha proporcionado un apoyo sólido a la hipótesis de que el capital social movilizado, en la forma de recursos sociales, contribuye de modo significativo a la consecución de estatus más allá de los recursos personales. Pero los datos disponibles parecen más ambiguos respecto a la proposición sobre fuerza de los vínculos. En opinión de Lin (2003) no existe una base empírica sólida para sostener que lazos más débiles permitan un mejor acceso a los recursos sociales. En cualquier caso, la tradición investigadora sobre capital social y logro de estatus ha generado una rica y abundante literatura científica que ha contribuido significativamente al desarrollo de la misma teoría de capital social y ha proporcionado datos empíricos detallados y variados para su verificación.

\section{Contribución del capital social al desa- rrollo del capital humano. La teoría norma- tivista de Coleman}

James Coleman comenzó a usar el concepto de capital social como una herramienta conceptual al servicio de su proyecto teórico de la introducir la estructura social dentro del paradigma de la acción racional. Su objetivo era neutralizar las premisas individualistas extremas del homo oeconomicus y al mismo tiempo dinamizar la visión excesivamente socializada del actor social predominante entre los sociólogos, integrando elementos de ambas tradiciones.

Coleman desarrolló su noción de capital social como complemento necesario de la noción de capital humano construida anteriormente como ampliación de la noción de capital físico.
Una diferencia fundamental entre el capital humano y el capital social es que el capital humano tiene su raíz en el individuo, mientras que el capital social emerge de las relaciones sociales. Pero tanto el uno como el otro pueden considerarse capitales en sentido estricto en tanto facilitan la actividad productiva, lo mismo que ocurre con el capital físico. Lo que les diferencia del capital físico es el grado de tangibilidad. Mientras el capital físico es totalmente tangible, el capital humano, plasmado en las habilidades y conocimientos adquiridos por un individuo, resulta menos tangible que aquél, y el capital social, en tanto existe en las relaciones entre las personas, lo es aún menos (Coleman, 1988, pp. 100-101).

El análisis de Coleman se centra en las funciones del capital social, al que definió como «una variedad de entidades» que tienen dos elementos en común: constituir «algún aspecto de las estructuras sociales» y facilitar la acción de los actores - personas o actores corporativos - dentro de la estructura» (1990, p. 302). $\mathrm{Su}$ foco se centra en las obligaciones y expectativas recíprocas, así como en las normas y sanciones que aseguran estas relaciones, tanto a nivel micro como macro (Svendsen y Svendsen, 2004, p. 251).

Entre los diferentes recursos que constituyen un capital social para los actores sociales, Coleman (1988, pp. 102-105) destaca tres: 1) las obligaciones, expectativas y fiabilidad de las estructuras, 2) el potencial de información inherente a las relaciones sociales y 3 ) las normas y sanciones eficaces. El capital social es un bien común que beneficia no sólo a los que lo producen sino a todos los miembros de la comunidad. Las redes densas y cerradas contribuyen a mantener el capital colectivo y conseguir su reproducción.

Una propiedad de las relaciones sociales de la que dependen las normas eficaces es lo que Coleman llama «cierre», término que denota la existencia de suficientes lazos entre cierto número de personas para garantizar las normas. El cierre de la estructura social es importante no sólo para la existencia de normas eficaces sino también para la fiabilidad de les estructuras sociales, otra forma de capital social que permite la proliferación de obligaciones y expectativas. Por ejemplo, para que los padres puedan fomentar el desarrollo cognitivo de los niños no 
sólo es necesario el capital humano sino también la conexión intergeneracional o «cierre» transgeneracional de la red social familiar. $\mathrm{La}$ lógica de la interconectividad se aplica también a las escuelas y otras organizaciones comunitarias (Coleman, 1988, pp. 105-108).

Las investigaciones empíricas de Coleman sobre el éxito o el fracaso escolar representan una rara oportunidad para observar la teoría del capital social en acción (Hagan et al., 1996, p. 371). Coleman (1990) analiza las consecuencias de la falta de capital social para la permanencia y éxito escolar de los adolescentes. El punto clave es que las relaciones socialmente estructuradas entre los miembros de las familias, escuelas o barrios son importantes fuentes de capital social que aumentan las probabilidades de éxito académico de los niños. Uno de los efectos más importantes de la carencia de capital social dentro de la familia es el abandono escolar. Cualquiera que sea el volumen del capital humano poseído por los padres resulta irrelevante para el desarrollo de los hijos, si no se completa con unas intensas interacciones entre ellos. Pero en la educación de los jóvenes no sólo tiene importancia el capital social de las familias sino también el capital social de la comunidad. Su relevancia se pone de manifiesto en que la frecuencia de los traslados familiares es la variable de mayor efecto global en el fracaso escolar, incluidas las del capital humano, el capital financiero y el capital social familiares (Coleman, 1988, pp. 108-113; Sampson, Morenoff y Gannon-Rowley, 1992).

Coleman sostiene que «el capital social tiene aspectos de bien público» (p. 116). Esta característica lo diferencia de otras formas de capital que suelen beneficiar a sus propietarios. Por eso considera que en las sociedades modernas las condiciones socioestructurales para generar este bien público, como la existencia de familias y comunidades consistentes, son cada vez menos frecuentes y que, si todo lo demás permaneciese igual, nos enfrentaríamos a un declive cuantitativo del capital humano en cada generación sucesiva. Para evitar esta consecuencia no deseada propone el diseño racional de nuevas instituciones como alternativa funcional a las organizaciones sociales voluntarias y espontáneas que han sido en el pasado la principal fuente del capital social para los jóvenes (pp. 116-118; 1992).
En síntesis, como puntos fuertes del concepto de capital social de Coleman cabe señalar la identificación de ciertos aspectos de la estructura social como recursos que los actores pueden usar para conseguir sus intereses; su contribución a la explicación de los diferentes resultados a nivel de actores individuales; el destacar la importancia del capital social para la adquisición del capital humano; la identificación de algunos de los mecanismos que lo generan, como el «cierre» estructural», y su utilidad para facilitar la transiciones de lo micro a lo macro. Como puntos débiles, se pueden destacar la imprecisión de la definición de capital social que nos ofrece Coleman, que, a diferencia de la elaborada por Bourdieu, no distingue con claridad los recursos de la habilidad para obtenerlos y tampoco entre motivaciones de los receptores y motivaciones de los donantes (Portes, 1998, p. 56).

\section{Algunos puntos débiles del la noción de capital social. El modelo de Portes y Sensen- brenner}

Alejandro Portes (2000) realizó una de las investigaciones más consistentes para poner a prueba la teoría del capital social de Coleman, especialmente su idea de que el «cierre» comunitario, que supone la implicación de otros adultos en la crianza y supervisión de los niños, amplia las capacidad supervisora y de apoyo de los padres a los hijos. Portes usó los datos del National Educational Longitudinal Study (NELS) que contiene buenas medidas empíricas de todas las variables de capital social que se considera afectan a la educación de los niños, además de indicadores fiables del logro escolar, para «comprobar el argumento de los fuertes efectos del capital social en el logro académico de los hijos de inmigrantes».

El principal resultado de la reconocida investigación de Portes fue que cuando se introducen controles para la edad y sexo de los estudiantes, el estatus socioeconómico de los padres, el conocimiento del inglés y el tiempo que llevan residiendo en los Estados Unidos, los efectos del capital social caen notoriamente y llegan a resultar insignificante tanto en el sentido de fiabilidad estadística como de importancia sustantiva (pp. 7-10).

Otra importante área de investigación para la teoría del capital social son los estudios de 
Portes sobre el empresariado étnico en comunidades inmigrantes de los Estados Unidos (Portes y Sensenbrenner, 1983; Portes, 1995). Partiendo de un análisis riguroso de los datos obtenidos, Portes sostiene que elevados niveles de capital social pueden ser «positivos» en tanto proporcionan a los miembros del grupo: acceso a recursos privilegiados, flexibles, disminución de los costes de las transacciones y apoyo psicológico; pero también pueden ser negativos en tanto impone elevadas exigencias particularistas a los miembros del grupo, restringiendo las expresiones y los avances individuales.

En opinión de Portes, es necesario tener en cuenta las diferentes funciones del capital social si se quiere evitar confusión y avanzar en su investigación. Es posible, por ejemplo, que el capital social en la forma de control social pueda chocar con el capital social en la forma de beneficios mediados por la red cuando éstos consisten en facilitar el evadir las normas existentes La capacidad de las autoridades para hacer cumplir las normas (control social) puede verse erosionada por la existencia de redes densas cuya función es precisamente facilitar la violación de estas normas en beneficio privado. Estos resultados paradójicos sugieren la necesidad de una mirada más precisa a los posibles ganadores y perdedores en las transacciones mediadas por el capital social. Los mismo que puede ser el origen de bienes públicos, como los destacados por Coleman, Laury y otros, el capital social también puede conducir a «males» públicos, como las familias de la mafia, la prostitución o la pandillas violentas (Portes, 1998, pp. 14-18)

El modelo de Portes y Sensenbrenner sobre los antecedentes y efectos del capital social ayuda mucho a identificar algunas de las condiciones de formación del capital social, enfatiza correctamente tanto los aspectos positivos como negativos en grupos concretos o comunidades localizadas y demuestra que las relaciones sociales necesitan ser dinámicas para acomodarse a un intercambio económico más complejo.

\section{Capital social, sentido cívico y participa- ción ciudadana. La teoría de Putnam}

Robert Putnam ha introducido un giro conceptual en el análisis sociológico del capital social al equipararlo con el sentido cívico y la participación ciudadana. Este politólogo define el capital social como: «las redes sociales y las normas asociadas de reciprocidad y fiabilidad» (2007, p. 37). En su opinión, la idea central de la teoría del capital social es sumamente sencilla: que las redes sociales y las normas de reciprocidad y confianza derivadas de ella poseen un valor, ante todo, para quienes participan en ellas. Las redes densas de interacción social parecen fomentar normas sólidas de reciprocidad generalizada que generan una confianza lubricadora de la vida social. En este sentido, el capital social está estrechamente relacionado con lo que algunos han llamado «virtud cívica» (Putnam, 2002, p. 14; 2003, pp. 13-14).

Lo fundamental de esta aproximación es tan «simple» como afirmar que las redes sociales tienen un valor, lo mismo que las herramientas (capital físico) o la instrucción (capital humano). Las redes sociales son valiosas, en primer lugar, para la gente que participa en ellas. Por ejemplo, el conseguir un empleo y los ingresos a lo largo de la vida están muy afectados por nuestras redes sociales, como han mostrado reiteradamente los sociólogos de la economía. Además, las redes sociales con frecuencia también tienen poderosas «externalidades», como la prevención del delito, lo que lo las hace incluso más interesantes (Putnam, 2007, pp. 137-138).

Putnam argumenta que el número de asociaciones cívicas y el grado de participación en ellas es el mejor indicador de la extensión del capital social en una sociedad. Estas asociaciones y la participación en ellas promueven y refuerzan las normas y la confianza colectivas, que son centrales para la producción y mantenimiento del bienestar colectivo (Putnam, 1993a, 1993b, 1995). En su obra El declive del capital social, Putnam (2003) plantea dos hipótesis: la primera, que cada vez es más evidente que las características de la sociedad civil afectan a la salud de nuestras democracias, comunidades y personas. La segunda, que las características más significativas de la sociedad civil varían de forma sistemática en el tiempo y el espacio.

Las redes sociales y las normas de reciprocidad concomitantes presentan muchas formas y usos diferentes. Los primeros estudios sobre 
el capital social se centraban en las asociaciones formales por razones de conveniencia metodológica. Pero este tipo de asociaciones no son más que una de las formas de capital social. Las asociaciones informales, como las actividades lúdicas, pueden ser más útiles que las formales para conseguir algunos objetivos valiosos. Las diferentes redes sociales pueden producir diferentes efectos: los amigos pueden mejorar la salud, mientras que los grupos cívicos fortalecen la democracia. Muchos datos sugieren que donde los niveles de capital social son más elevados, los niños crecen más sanos, seguros y mejor educados, la gente vive más, vive más feliz y la democracia y la economía funcionan mejor (Putnam, 2000, 2003)

Esto no significa que los efectos externos del capital social sean siempre beneficiosos. El hecho de que las redes pueden aumentar mucho la capacidad para conseguir objetivos, no garantiza que lo que se consigue mediante las redes sea socialmente beneficioso, como sucede en el caso de Al Qaeda, por lo demás un excelente ejemplo de capital social. Como cualquier otra forma de capital, el capital social puede emplearse para fines antisociales. Es por ello importante considerar "cómo se pueden maximizar los efectos beneficiosos del capital social - apoyo mutuo, cooperación, confianza institucional, eficacia - y minimizar los perjudiciales - sectarismo, etnocentrismo, corrupción» (Putnam, 2002, p. 19).

Para ello resulta especialmente pertinente la distinción establecida por algunos investigadores entre dos variedades del capital social: el capital social vinculante (bonding) y el capital social puente (bridging). El primero une a personas que comparten algunas características socialmente relevantes como la etnia, el género, la clase social o la edad, mientras que el capital social que tiende puentes se refiere a las redes sociales que incluyen a personas con características sociales diferenciales (Putnam 2003, pp. 19-20). Ciertas formas de capital social o redes sociales tienden a reforzar las identidades excluyentes y los grupos homogéneos, mientras que otras también miran hacia fuera $\mathrm{e}$ incluyen a personas de diferentes estratos sociales. Aunque en la práctica resulta difícil establecer divisiones tan nítidas, las categorías de inclusión y exclusión nos sirven para distinguir dos tipos de capital social que no son intercam- biables (Putnam, 2002, pp. 20-22). Con frecuencia se da por supuesto que el capital social puente y el capital social vinculante se correlacionan inversamente en un tipo de relación de suma cero. Putnam, por el contrario, opina que un elevado capital social vinculante puede ser compatible con algo de capital punte, y un bajo capital social vinculante con bajo capital puente. Aunque se trata de una cuestión empírica, Putnam considera que este planeamiento teórico ayuda a clarificar la relación.

El aumento de la heterogeneidad social es «uno de los desafíos más importantes a los que se enfrentan las sociedades modernas y al mismo tiempo una de nuestras oportunidades más significativas». Recientemente Putnam ha comenzado a explorar las implicaciones que tiene para el capital social esta transición hacia una sociedad más multicultural (Putnam, 2007, p. 137). En su opinión, el aumento de la inmigración y diversidad no sólo no son inevitables sino que a largo plazo son deseables. Como demuestra la historia de los Estados Unidos, «la diversidad étnica es, en conjunto, un activo social importante». En el medio a largo plazo las sociedades inmigrantes exitosas crean nuevas formas de solidaridad social y diluyen los efectos negativos de la diversidad construyendo nuevas identidades más amplias. Sin embargo, «en el corto a mediano plazo, la inmigración y la diversidad étnica desafían la solidaridad social e inhiben el capital social». Por ello, «el desafío central para las sociedades modernas diversificadas es crear un nuevo sentido más amplio del "nosotros"» (pp. 138-139).

A pesar de su indudable éxito, el planteamiento de Putnam ha recibido importantes críticas. Las principales se han centrado en la cuestión de si el voluntariado y el espíritu cívico han disminuido en Norteamérica, como sostiene este autor, o los que ocurre más bien es que está adquiriendo nuevas formas Algunos autores consideran que al centrarse en el papel de la sociedad civil en la construcción del capital social, Putnam desplaza a una posición secundaria al Estado, actor fundamental en la política moderna (Ríos y Ríos, 1999). Skocpol enfatiza un sesgo de clase no reconocido en la tesis de Putnam, quien, tal vez de modo no intencionado, «ignora en gran medida las dinámicas interclasistas y organizativas por las cuales en realidad se forman y persisten las 
asociaciones cívicas, o decaen y se deshacen» (1996, pp. 21-22). En opinión de Portes el problema más fundamental del argumento de Putnam es su «circularidad lógica» (1998, p. 19).

En cualquier caso, las obras de Putnam, lo mismo que las de Coleman, representan una visión del capital social como un activo colectivo y enfatiza su carácter de bien público. Estos activos colectivos están a disposición de todos los miembros del grupo, sea éste un grupo social o una comunidad, y sean quienes sean los miembros que en realidad promuevan, sostengan o contribuyan a tales recursos. Como bien público, el capital social depende de la buena voluntad de los miembros individuales para hacer los esfuerzos que requiere.

La influyente investigación de Coleman sobre la educación y especialmente la de Robert Putnam sobre participación ciudadana y desempeño institucional, han proporcionado inspiración a la mayoría del trabajo que se está realizando en campos diferentes: 1) familias y problemas de comportamiento juvenil; 2) escolarización y educación; 3 ) vida comunitaria («virtual» y cívica); 4) trabajo y organizaciones; 5) democracia y «gobernanza»; 6) acción colectiva; 7) salud pública y temas medioambientales; 8) delincuencia y violencia, y 9) desarrollo económico (Woolcok 2000, p. 2).

\section{El capital social como instrumento de de- sarrollo. La síntesis de Woolcock}

Una de las aplicaciones más fecundas de la teoría del capital social es la que impulsó Michael Woolcock, quien vio en la idea de capital social la posibilidad de incorporar a una teoría interdisciplinar del desarrollo la contribución de la sociedad civil como mediadora entre los estados y los mercados, algo que - según este autor- habían descuidado la teorías contemporáneas de desarrollo, tanto las del sistema mundial como las neoclásicas. Haciéndose eco de la observación de Putnam (1993a) sobre las alarmantes deficiencias de capital social en los países en vías de desarrollo, Woolcock se propuso elaborar «un marco coherente para incorporar el capital social en la teoría política del desarrollo» (1998, p. 154).

Woolcock integró en un marco conceptual unificado dos perspectivas de la llamada «nueva sociología del desarrollo económico», el en- foque micro de los estudios del empresariado étnico (Portes y Sensenbrennner, 1993) y el enfoque macro de los estudios institucionales comparativos de las relaciones entre el estado y la sociedad (Evans, 1989; Evans, Rueschemeyer y Skocpol, 1985). Las limitaciones conceptuales y empíricas de ambos tipos de estudios surgieren la necesidad de un modelo más amplio y más dinámico que abarque ambos dominios.

La base de la síntesis de Wollcock es la centralidad de los conceptos de arraigo (embeddedness) y autonomía (autonomy), que corresponden a las dos formas de capital social diferentes, pero complementarias, que vimos anteriormente, el capital social vinculante y el capital social puente. El sentido en el que los investigadores de la nueva sociología del desarrollo emplean los términos «arraigo» y «autonomía» difiere según se trate del nivel micro o macro. El arraigo a nivel micro se refiere a los lazos intracomunitarios, mientras que a nivel macro ser refiere a las relaciones estado-sociedad. En cuanto al término autonomía, a nivel micro designa las redes extra-comunitarias, mientras que a nivel macro se refiere a la credibilidad institucional. Esto no crea un problema irresoluble, pero sugiere que cualquier síntesis del capital social tal como se ha desarrollado a los niveles micro y macro ha de integrar cuatro formas distintas.

Las diferentes combinaciones de estas cuatro dimensiones del capital social, integración $\mathrm{y}$ vinculación a nivel micro e integridad y sinergia a nivel macro, pueden explicar un rango de resultados de desarrollo, desde el «individualismo anárquico» (la ausencia de esas cuatro dimensiones) en un extremo a la autonomía altruista (la presencia de las cuatro) en el otro. Una misma dimensión del capital social puede así servir a propósitos de desarrollo muy diferentes cuando se combina con otras formas. Las relaciones sociales en general constituyen un recurso único, pero muy problemático en cuanto a las consecuencias positivas para el desarro1lo, donde surgen importantes dilemas teóricos y políticos. Woolcock distingue entre dilemas de abajo a arriba (bottom-up) y dilemas de arriba abajo (top-down).

Las iniciativas de abajo arriba de desarrollo son aquellas que emergen o tienen lugar a nivel local. El desarrollo de abajo arriba funcio- 
na típicamente en y a través de las relaciones sociales entre gente que comparte lazos de vecindad, étnicos, religiosos o familiares. En este caso se presenta un dilema, pues más no significa mejor. Donde la confianza generalizada se extiende sólo a los miembros de la familia y parientes se puede producir lo que Edward Banfield llama «familismo amoral». Esta situación se caracteriza por un «exceso de comunidad» construida sobre lealtades étnicas y vinculaciones familiares tan intensas que sus miembros son disuadidos de progresar económicamente, moverse geográficamente y comprometerse en una resolución amistosa de los conflictos con los extraños. El «familismo amoral» se caracteriza por la presencia de integración social, pero con ausencia de vinculación. En el polo opuesto hallamos la situaciones de «individualismo amoral», donde no hay ni confianza familiar ni confianza generalizada, donde el propio interés limitado impregna literalmente toda la actividad social y económica, y donde los miembros de una comunidad están aislados de todas las formas de redes sociales cohesivas, bien por discriminación u otras circunstancias, como puede ser la situación de los sin techo (Anderson, 1923).

Para que las comunidades pobres logren desarrollarse necesitan combinar la integración de los vínculos fuertes y débiles. Los lazos intracomunitarios pueden ser muy beneficiosos en la medida en que se completan con otros vínculos fuera de la comunidad (Alba y Nee, 2005). Los beneficios iniciales de una intensa integración intracomunitaria deben dejar paso con el tiempo a amplias vinculaciones extracomunitarias. Demasiado o demasiado poco de cualquiera de estas dimensiones en un momento dado mina el avance económico. Las aportaciones de los teóricos clásicos y de los estudios contemporáneos de la pobreza urbana y del empresariado étnico sugieren que los grupos de negocios en las comunidades pobres necesitan forjar y mantener vínculos que trasciendan la comunidad.

Hay un segundo tipo de dilemas que se producen a nivel macro: de arriba abajo. Las dinámicas internas y el desarrollo de grupos económicos en comunidades pobres no ocurre aisladamente, sino más bien en el contexto de una historia y un marco regulativo concretos que pueden fortalecer o minar la capacidad de grupos indepen- dientes en la sociedad civil para organizarse en torno a sus propios intereses colectivos. Tales grupos, a su vez, pueden jugar un rol importante en la configuración de las políticas y actuaciones del gobierno. Centrase en las condiciones que fomentan la complementariedad y la cooperación eficaz entre el Estado y la sociedad civil, entre los sectores público y privado contribuye a forjar un camino para la teoría del desarrollo entre los modelos socialistas rígidos, las prescripciones comunitarias aislacionistas y las doctrinas simplistas del mercado libre. Este camino requiere una comprensión más sofisticada del rol de las relaciones estado sociedad en el desarrollo.

Un rango de resultados de desarrollo es posible, dependiendo de la combinación que prevalezca de la capacidad organizativa del Estado y de su compromiso y receptividad a las propuestas de la sociedad civil. En un extremo estarían los llamados «estados fallidos», estrechamente relacionados con lo que Evans (1998) llama «estados predatorios». Para que se produzca el desarrollo es necesaria pero no suficiente la integridad organizativa. Esta integridad puede resultar improductiva si, además, no existe una sinergia en las relaciones entre Estado y sociedad y lo que vale para estas relaciones generalmente también es válido para todas las formas de desarrollo de arriba abajo.

El marco del capital social diseñado por Woolcock no sólo identifica obstáculos concretos para el desarrollo, sino que también aclara varias oportunidades importantes para intervenciones políticas positivas. Los resultados del desarrollo dependen de cómo se resuelvan los dilemas sociales básicos a nivel micro y macro. Se obtienen resultados positivos en la medida en que las relaciones arraigadas y autónomas mantienen un equilibrio en ambos niveles. Para obtener resultados óptimos de desarrollo es necesario que la gente quiera y pueda recurrir a cultivar cuatro dimensiones del vínculo social: intracomunitaria, entre la sociedad civil y las instituciones a nivel macro, e interinstitucional.

El capital social proporciona un fecundo diseño conceptual y político que permite ir más allá de las exhaustas teorías de la modernización y del sistema mundial y hacer contribuciones potencialmente importantes a cuestio- 
nes de desarrollo económico, contribuciones que en algunos aspectos completan las aproximaciones económicas ortodoxas y en otros las desafían. El mayor mérito del capital social, sin embargo, es que proporciona un punto de partida razonable para una aproximación comprensiva multidisciplinar e interdisciplinar para algunos de los temas sociopolíticos más urgentes de nuestro tiempo (Woolcok, 1998, pp. 162-188). Este autor (Woolcock, 2000) identifica cuatro aproximaciones diferentes a las relaciones entre capital social y desarrollo económico:

1. La perspectiva comunitaria: equipara el capital social con organizaciones a nivel local, como asociaciones, clubes y grupos cívicos. Esta perspectiva ha hecho importantes contribuciones al análisis de la pobreza, enfatizando la centralidad de los lazos sociales para ayudar a los pobres a gestionar el riesgo y la vulnerabilidad. Pero los que siguen este enfoque han ignorado con frecuencia los importantes inconvenientes que pueden presentarse cuando el capital social vinculante no se compensa con alguna medida de capital social puente, como han señalado Portes y Landolt (1996).

2. La perspectiva de redes: enfatiza la importancia tanto de las asociaciones horizontales como de las verticales, como grupos comunitario y empresas. Este enfoque platea -a la investigación e intervención en temas de desarrollo - la necesidad de identificar las condiciones bajo las cuales las comunidades pobres pueden obtener un equilibrio entre los fuertes vínculos intragrupo y el acceso a las instituciones formales y a una reserva más diversificada de capital social puente, condición indispensable para desarrollo equilibrado y sostenido. Uno de sus puntos más débiles es que no incorpora en su análisis a las instituciones a nivel macro, como el Estado y su interacción con las comunidades locales.

3. La perspectiva institucional: la vitalidad de las redes comunitarias y de la sociedad civil en general es, en cierta medida, el producto de un entorno político, legal e institucional. A diferencia de las dos perspectivas anteriores, que en gran medida tratan el capital social como una variable independiente que produce varios «bienes» o «males», el enfoque institucional pone el énfasis en el capital como una variable dependiente. Paradójicamente, el verdadero punto fuerte de este enfoque, el abordar las preocupaciones macropolíticas, es también su debilidad, en tanto carece de un componente micro.

4. La perspectiva de la sinergia: pretende integrar las perspectivas de redes e institucional (World Development, 1990; Evans, 1996; Woolcock, 1998; Woolcock y Narajaj, 2000). Este enfoque enfatiza que el desarrollo inclusivo tiene lugar cuando los representantes del Estado, el sector empresarial y la sociedad civil establecen foros comunes para identificar y perseguir metas comunes. El capital social se trata en este caso como una variable intermedia. Este enfoque sugiere tres tareas centrales para los teóricos, investigadores y responsables políticos: la primera, identificar la naturaleza y amplitud de las relaciones que caracterizan a una comunidad. La segunda, desarrollar estrategias institucionales basadas en la comprensión de estas relaciones sociales, concretamente extender el capital social vinculante y puente. La tercera, identificar vías y medios por los que las manifestaciones positivas del capital social, amplia cooperación, confianza, y eficiencia institucional, compensen las negativas, como el sectarismo, el aislacionismo y la corrupción (Woolcok, 2000, pp. 6-14).

\section{Desigualdad y capital social}

La desigualdad social despende no sólo de la distribución desigual de los recursos económicos, sino del acceso diferencial a otros tipos de capital. Como ha observado Pierre Bourdieu: «De todas las distribuciones, una de las más desiguales $\mathrm{y}$, sin duda, en cualquier caso, la más cruel, es la del capital simbólico, es decir, de la importancia social y las razones para vivir» (1999, p. 317).

Un tema crítico para la investigación del capital social es en qué medida la desigualdad de capital social contribuye a la desigualdad social. Tal investigación supone, según Lin (2003), el análisis de dos procesos desde la perspectiva del capital: el déficit de capital y el déficit de rendimiento. ¿En qué medida y por qué razones, de inversión u oportunidad, los diferentes grupos sociales han llegado a poseer diferente cantidad o calidad de capital? ¿Cuáles son las diferentes estrategias de movilización, esfuerzos de los agentes o respuestas ins- 
titucionales que hacen que una determinada calidad o cantidad de capital genere beneficios para diferentes grupos sociales

El acceso diferencial al capital social merece mucha mayor atención investigadora. Los grupos sociales tienen un acceso desigual al capital social debido a sus posiciones estructurales y a sus redes. Para que los desfavorecidos consigan mejorar estatus o un desarrollo sostenido necesitan acciones estratégicas que les permitan acceder a recursos más allá de sus círculos sociales habituales, desarrollando vínculos fuera del propio barrio o comunidad y de los compañeros de trabajo o a través de las fronteras étnicas, en el caso de las minorías étnicas.

\section{Referencias bibliográficas}

Alba, Richard y Nee, Victor (2005). Remaking the American Mainstream. Cambridge MA: Harvard University Press.

Anderson, Nels (1923). The Hobo; the Sociology of the Homeless Man. Chicago: University of Chicago Press.

Bourdieu, Pierre (1980). Le capital social. Notes provisoires. En Actes de la recherche en Sciences Sociales, 31, 2-3.

Bourdieu, Pierre (1986). The forms of capital. En J. Richardson (ed.), Handbook of Theory and Research for the Sociology of Education (pp.241-258). Nueva York: Greenwood.

Bourdieu, Pierre (1989). La «noblesse d'État». Grandes écoles et sprit de corps. París: Minuit.

Bourdieu, Pierre (1999). Meditaciones Pascalianas. Barcelona: Anagrama.

Bourdieu, Pierre (2003). Las estructuras sociales de la economía. Barcelona: Anagrama.

Burt, Ronald (1992). Structural holes. Cambridge, MA: Harvard University Press.

Coleman, James S. (1988). Social Capital in the Creation of Human Capital. American Journal of Sociology, 94, 95-105.

Coleman, James S. (1990). Foundations of Social Theory, Cambridge, MA: Harvard University Press.

Coleman, James S. (1993). The Rational Reconstitution of Society. American Sociological Review, 58, 1-15.

Evans, Peter (1989). Predatory, developmental and other apparatuses: a comparative political economy perspective on the third world sate. Sociological Forum, 4 (4/4), 561-587.

Evans, Peter, Rueschemeyer, Dietrich y Skocpol,Theda (1985). Bringing the State Back In. Cambridge: Cambridge University Press.

Fukerson, Gregory M. y Thomson, Gretchen H. (2008). The Evolution of a Contested Concept: A Meta-Analysis of Social Capital. Sociological Inquiry 78 (4), 536-557.

Granovetter, Marks (1973). The Strength of Weak Ties. American Journal of Sociology, 78 (6), 1360-1380.

Granovetter, Marks (1974). Getting a Job: A Study of Contacts and Careers. Cambridge, MASS: Harvard University Press.

Granovetter, Marks (1983). The Strength of Weak Ties: A Network Theory Revisited. Sociological Theory, 1, 201-233.

Lin, Nan (1982). Social Resources and Instrumental Action. En P.V. Marsden (ed.), Social Structure and Network Analysis (pp.131-145). Beverly Hills, CA: Sage.

Lin, Nan (2003). Social capital. A Theory of Social Structure and Action, Cambridge: Cambridge University Press.

Lin, Nan (2008). A network theory of social capital. En Dario Castiglione, The Handbook on social capital, Oxford: Oxford University Press.

Lin, N., Dayton, P. y Greenwald, P. (1978). The urban communication network and social stratification: A «small world experiment». En B. D. Ruben (ed.), Communication yearbook 1 (pp. 107-119). New Brunswick: Transaction Books.

Lin, Nan, W, Ensel, Walter y Vaughn, John (1981). Social Resources and Strength of Ties: Structural Factors in Occupational Attainment. American Sociological Review, 46 (4), 393-405.

Portes, Alejandro (ed.) (1995). Economic sociology and sociology of immigration. Nueva York: Russell Sage Foundation. 
Portes, Alejandro (1996). The new second generation: segmented assimilation and its variants. Nueva York: Russell Sage Foundation.

Portes, Alejandro (1998). Social Capital: Its Origins and Applications in Moderns Sociology. Annual Review of Sociology, 24, 1-24.

Portes, Alejandro (2000). The two meanings of Social Capital. Sociological Forum, 15 (1), 1-15.

Portes, Alejandro y Sensenbrenner, (1993). Embeddedness and immigration: notes on the social determination of economic action. American Journal of Sociology, 98 (6), 1320-1350.

Portes, Alejandro y Landolt, Patricia. (1996). The downside of social capital. The American Prospect, 26, 18-21.

Putnam, Robert, D. (1993 a). The Prosperous Community: Social Capital and public life. American Prospect, 13, 35-42.

Putnam, Robert, D. (1993 b). Making Democracy Work Civic Traditions in Modern Italy. Princeton: Princeton University Press.

Putnam, Robert, D. (enero, 1995). Bowling Alone: American`s Declining Social Capital. Journal of Democracy, 6 (1), 65-78.

Putnam, Robert, D. (2002). Solo en la bolera. Colapso y resurgimiento de la comunidad norteamericana. Barcelona: Galaxia Gutenberg/Círculo de Lectores.

Putnan, Robert D. (ed.) (2003). El declive del capital social. Un estudio internacional sobre las sociedades y el sentido comunitario. Barcelona: Galaxia Gutenberg y Círculo de Lectores.

Putnan, Robert D (2007). E Pluribus Unum: Diversity and Community in the Twenty first Century. The 2006 Johan Skyttte Prize Lecture. Scandinavian Political Studies, 30 (2), 137-168.

Ríos Cazares, Alejandra y Ríos Figueroa, Julio (1999). Capital Social y democracia: una revisión crítica de Robert Putnam. Política y Gobierno, 6 (2), 513-528.

Sampson, Robert, J., Morenoff, J. y Gannon-Rowley, T. (1992).Assessing Neighbourhood Effects: Social Processes and New Directions in Research. Annual Review of Sociology, 28, 443-478.

Skocpol, Theda (1996).Unravelling from above. American Prospect, 25, 20-25.

Smart, Alan (1993). Gifts, Bribes, and Guanaxi: A Reconsideration of Bourdieu`s Social Capital. Cultural Anthropology, 8 (39), 388-408.

Svendsen, Gunnar Lind H. y Svendsen, Gert T. (2004). On the welth of nations: Bourdieueconomics and social capital. En David, Swarttz, L. Zolberg y L. Vera, (eds.), After Bourdieu, Influence, Critique, Elaboration. Dordrecht: Kluwer Academic Press.

Woolcock, Michael (1998). Social Capital and Economic Development: Toward a Theoretical Synthesis and Policy Framework. Theory and Society, 27, 151-208.

Woolcock, Michael y Nararaj, Deepa (2000).Social Capital: Implications for Development Theory, Research and Policy. World Bank Research Observer, 15 (2). 